\title{
Biinvariant Operators on Nilpotent Lie Groups
}

\author{
David Wigner * \\ The University of Michigan, Department of Mathematics Ann Arbor, \\ Michigan 48109, USA
}

The purpose of this article is to prove $P$-convexity for biinvariant differential operators on connected simply connected nilpotent Lie groups. More precisely, we show that for any compact subset $K$ of a connected simply connected nilpotent Lie group $N$, and for any non-zero biinvariant differential operator $P$ on $N$, there is a compact subset $L \supset K$ with the property that whenever the support of $P u$ is contained in $L$ for a $C^{\infty}$ function of compact support $u$ on $N$, then the support of $u$ is contained in $L$. I am grateful to M. Duflo, to A. Cerezo, and to F. Rouvière for several helpful discussions.

Solubility properties of biinvariant operators have been considered by several authors. S. Helgason [6] proves local solvability of biinvariant operators on semisimple Lie groups. Rais [8] proves the existence of a fundamental solution for a biinvariant operator on a connected simply connected nilpotent Lie group. Duflo and Rais [4] prove the local solvability of biinvariant operators on a solvable Lie group and Rouvière [9] proves semi-global solvability for biinvariant operators on simply connected solvable groups. Finally, Duflo [3] proves local solvability of biinvariant operators on any Lie group whatsoever.

Semi-global solvability is in general false even for noncompact simple groups as was demonstrated by A.Cerezo and F. Rouvière [2]. Finally, even local solvability of left invariant operators is frequently false as was shown by L. Hormander, c.f. [6] and independently by A. Cerezo and F. Rouvière [1]. From our result and that of Rais [8] or Rouvière [9], we conclude the global solvability of biinvariant operators on simply connected nilpotent Lie groups, i.e. that for any $C^{\infty}$ function $f$ and nonzero biinvariant operator $P$ on a simply connected nilpotent Lie group $N$, there exists a $C^{\infty}$ function $u$ on $N$ such that $P u=f$. For simply connected abelian Lie groups, this reduces to the theorem of Malgrange and Ehrenpreis that constant coefficient differential operators on $R^{n}$ are globally solvable, c.f. [11]. Thus our Theorem 2 can be regarded as a generalization of the Malgrange-Ehrenpreis theorem.

Henceforward $N$ will denote a connected simply connected nilpotent Lie group, and $\mathfrak{N}$ its Lie algebra. We write exp: $\mathfrak{N} \rightarrow N$ for the exponential map of $\mathfrak{N}$ 
onto $N$, which is known to be an analytic diffeomorphism and $\log : N \rightarrow \mathfrak{M}$ will denote the analytic diffeomorphism inverse to exp. We recall that the center of $N$ is connected and simply connected. Since a connected and simply connected abelian Lie group has a natural translation invariant convex structure, we may define a subset $S$ of $N$ to be $C$-convex if its intersection with every coset of the center $C(N)$ of $N$ is convex or in other words if $x^{-1}(S \cap x C(N))$ is a convex subset of $C(N)$ for every $x \in N$.

The support of a function will mean the set of points where it is non-zero (this is a departure from the usual usage). When we say a function has compact support, we mean that its support is contained in some compact set (this is the usual usage). Supp $f$ will denote the support of $f$, a complex valued $C^{\infty}$ function. $Z$ will denote a central one parameter subgroup of $N$, and $z$ will denote a generator of the Lie algebra of $Z$. Thus $z$ is a biinvariant vector field on $N$. We denote a Haar measure on $Z$ by $d \mu(z)$. If $f$ is a $C^{\infty}$ function of compact support on $N$ then $\tilde{f}$ will denote the function on $N / Z$ defined by $\tilde{f}(x Z)=\int_{Z} f(x z) d \mu(z)$. We note that if $f \equiv 0$ then there is a $C^{\infty}$ function $u$ of compact support on $N$ such that $z u=f$. We denote the natural projection of $N$ onto $N / Z$ by $\pi$ and remark that the inverse image under $\pi$ of a $C$-convex subset of $N / Z$ is $C$-convex. $P$ will denote a biinvariant differential operator on $N$. We shall identify the algebra of left invariant differential operators on $N$ with the complexified universal envelopping algebra $U(\mathfrak{N})$ of $\mathfrak{N}$. Following Trèves [10], we say that a subset $S$ of $N$ is $P$-full if whenever $P u=f$ is a $C^{\infty}$ function of compact support whose support is contained in $S$, and $u$ has compact support, then the support of $u$ is contained in $S$. Since $P$ is biinvariant, any (left or right) translate of a $P$-full set is $P$-full. A $C$-convex set is $z$-full for any biinvariant vector field $z$.

A $C^{\infty}$ function $f$ on $N$ will be called $Z$-invariant if $f(x z)=f(x)$ for all $x \in N$ and all $z \in Z$. When $P$ is biinvariant differential operator on $N$, then the action of $P$ on $Z$-invariant functions defines a differential operator on $N / Z$, denoted $\tilde{P}$. By "differentiating under the integral", we have $\widetilde{P u}=\tilde{P} \tilde{u}$ for a $C^{\infty}$ function $u$ of compact support on $N$.

We begin with some preparatory lemmas.

Lemma 1. Let $Z$ be a central one parameter subgroup of $N$, and let $z$ be a generator of the Lie algebra of $Z$. Let $D$ be a left invariant differential operator on $N$ which annihilates all $Z$-invariant functions. Then $D=D_{1} \circ z$ where $D_{1}$ is some left invariant operator on $N$. If $D$ is biinvariant, so is $D_{1}$.

Proof. Let $\tilde{x}_{1}, \tilde{x}_{2}, \ldots, \tilde{x}_{n}$ be a basis of the Lie algebra of $N / Z$ and let $x_{1}, x_{2}, \ldots, x_{n}, z$ be a basis of the Lie algebra $\mathfrak{N}$ of $N$ such that the projection of $x_{i}$ onto the Lie algebra of $N / Z$ is $\tilde{x}_{i}$. The Poincaré-Birkhoff-Witt theorem implies that monomials of the form $x_{1}^{a_{1}} x_{2}^{a_{2}} \ldots x_{n}^{a_{n}} z^{k}$, with $a_{1}, a_{2}, \ldots, a_{n}$, $k$ nonnegative integers form a basis of the vector space of left invariant differential operators on $N$ so that we may write

$$
D=\sum_{\left(a_{1} \ldots a_{n}, k\right)} C_{\left(a_{1}, a_{2} \ldots a_{n}, k\right)} x_{1}^{a_{1}} \ldots x_{n}^{a_{n}} z^{k}
$$

where the sum runs over $(n+1)$-tuples of non-negative integers, and all but finitely many of the $C_{\left(a_{1} \ldots a_{n}, k\right)}$ are zero. The action of $D$ on $Z$-invariant functions 
defines an operator $\tilde{D}$ on $N / Z$ and we have

$$
0=\tilde{D}=\sum_{\substack{\left(a_{1} \ldots a_{n}, k\right) \\ k=0}} C_{\left(a_{1}, \ldots, a_{n}, k\right)} \tilde{x}_{1}^{a_{1}} \tilde{x}_{2}^{a_{2}} \ldots \tilde{x}_{n}^{a_{n}}
$$

The Poincare-Birkhoff-Witt theorem now implies that $C_{\left(a_{1}, a_{2}, \ldots, a_{n}, k\right)}=0$ whenever $k=0$ so that we may write

$$
\begin{aligned}
D & =\sum_{\substack{\left(a_{1} \ldots a_{n}, k\right) \\
k>0}} C_{\left(a_{1} \ldots a_{n}, k\right)} x_{1}^{a_{1}} \ldots x_{n}^{a_{n}} z^{k} \\
& =\sum_{\substack{\left(a_{1} \ldots a_{n}, k\right) \\
k>0}} C_{\left(a_{1} \ldots a_{n}, k\right)} x_{1}^{a_{1}} \ldots x_{n}^{a_{n}} z^{k-1} \circ z=D_{1} \circ z
\end{aligned}
$$

where $D_{1}=\sum_{\substack{\left(a_{1} \ldots a_{n}, k\right) \\ k>0}} C_{\left(a_{1} \ldots a_{n}, k\right)} x_{1}^{a_{1}} \ldots x_{n}^{a_{n}} z^{k-1}$.

Now suppose $D$ is biinvariant and let $\rho_{g}$ denote right translation by $g \in N$. We then have

$$
D_{1} \circ z=D=\rho_{g} D=\rho_{g}\left(D_{1} \circ z\right)=\left(\rho_{g} D_{1}\right) \circ\left(\rho_{g} z\right)=\rho_{g} D_{1} \circ z
$$

so that $\left(\rho_{g} D_{1}-D_{1}\right) \circ z=0$.

But the universal envelopping algebra has no divisors of zero and $z \neq 0$ so $\rho_{g} D_{1}-D_{1}=0$ and $\rho_{g} D_{1}=D_{1}$. Therefore $D_{1}$ is biinvariant.

Lemma 2. If $u$ has compact support on $N$, then $\pi \operatorname{supp} \approx u=\pi \operatorname{supp} u$.

Proof. Since $\operatorname{supp} z u \subset \operatorname{supp} u$, clearly $\pi \operatorname{supp} z u \subset \pi \operatorname{supp} u$. Now let $x \in \operatorname{supp} u$ so that $\pi(x) \in \pi \operatorname{supp} u$. Define

$$
\phi: Z \rightarrow \mathbb{C} \quad \text { by } \phi(z)=u(x z) .
$$

$\phi$ is a non-zero function on $Z$ of compact support so $z \phi$ is non-zero of compact support on $Z$. But $z u(x z)=z \phi(z)$ so $z u$ is not identically zero on $x Z$ so $\pi(x) \in \operatorname{supp} z u$.

Proposition 1. If $K$ is a $P$-full set in $N / Z$, then $L=\pi^{-1}(K)$ is a $P$-full set in $N$.

Proof. Let $b$ be a smooth function of compact support on $Z$ with $\int_{z} b(z) d \mu(z)=1$.

Let $\sigma: N / Z \rightarrow N$ be a continuous map satisfying $\pi \circ \sigma=\operatorname{Id}_{N / Z}$.

For any complex function $f$ of compact support on $N$ define $f^{*}: N \rightarrow \mathbb{C}$ by

$$
f^{*}(x)=f(x)-\tilde{f}(\pi(x)) \cdot b\left(x \cdot(\sigma(\pi(x)))^{-1}\right) .
$$

Then

$$
\begin{aligned}
\int f^{*}(x z) d \mu(z) & =\int f(x z) d \mu(z)-\int \tilde{f}(\pi(x z)) \cdot b\left(x z \cdot(\sigma(\pi(x z)))^{-1}\right) d \mu(z) \\
& =f(\pi(x))-\int \tilde{f}(\pi(x)) \cdot b\left(x z(\sigma(\pi(x)))^{-1}\right) d \mu(z) \\
& =f(\pi(x))-f^{f}(\pi(x)) \int b\left(x z(\sigma(\pi(x)))^{-1}\right) d \mu(z) \\
& =0
\end{aligned}
$$


Therefore, there is a function $f^{\natural}$ of compact support on $N$ satisfying $z f^{\natural}=f^{*}$. Let $f$ be a function of compact support on $N$ whose support is contained in $L$. Let $P u=f$, where $u$ is also a function of compact support. Define inductively $u_{0}$ $=u$ and $u_{n+1}=u_{n}^{\natural}$. We have $\pi \operatorname{supp} P u_{n+1}=\pi \operatorname{supp} P u_{n}^{\natural}=\pi \operatorname{supp} z P u_{n}^{\natural}=\pi \operatorname{supp} P$ $\approx u_{n}^{\natural}=\pi \operatorname{supp} P u_{n}^{*} \subset \pi \operatorname{supp} P u_{n} \cup \operatorname{supp} \tilde{u}_{n}$. If $\pi \operatorname{supp} P u_{n} \subset K$ then supp $\tilde{u}_{n} \subset K$ since then $K \supset \operatorname{supp} \tilde{P} u_{n}=\operatorname{supp} \tilde{P} \tilde{u}_{n}$ and $K$ is $\tilde{P}$-full. Therefore if $\pi$ supp $P u_{n} \subset K$ then $\pi \operatorname{supp} P u_{n+1} \subset K$ and also supp $\tilde{u}_{n} \subset K$. But $\pi \operatorname{supp} P u_{0} \subset K$ so by induction on $n$ we have $\pi \operatorname{supp} P u_{n} \subset K$ and supp $\tilde{u}_{n} \subset K$, for all $n$.

Furthermore $\pi \operatorname{supp}\left(u_{n}^{*}-u_{n}\right) \subset \operatorname{supp} \tilde{u}_{n} \subset K$ and $\pi \operatorname{supp} u_{n+1}=\pi \operatorname{supp} z u_{n+1}$ $=\pi \operatorname{supp} u_{n}^{*}$.

Suppose now that $x \notin L$. On the set $x Z$ we have

$$
u_{n}^{*}(x z)=u_{n}(x z) \quad \text { and } \quad z u_{n+1}(x z)=u_{n}^{*}(x z)
$$

since $x Z$ is disjoint from $L=\pi^{-1}(K)$. So on $x Z$ we have $z u_{n+1}=u_{n}$ and $z^{n} u_{n}=u_{0}$ $=u$. Therefore, if $\phi_{n}(z)=u_{n}(x z)$, then $\phi_{0}$ is a function of compact support on $Z$ such that for arbitrary $n$ there exists a function $\phi_{n}$ of compact support on $Z$ such that $z^{n} \phi_{n}=\phi_{0}$. Applying the Fourier transform to $\phi_{0}$, we see that $\hat{\phi}_{0}$ is a real analytic function on the dual $\hat{Z}$ of $Z$ with a zero of arbitrary high order at $0 \in \hat{Z}$. Therefore $\hat{\phi}_{0} \equiv 0$ and $\phi_{0} \equiv 0$. Therefore $u(x z)=0$ for all $z$ and $x \notin \operatorname{supp} u$. QED.

Theorem 1. Let $P$ be a non-zero biinvariant differential operator on a simply connected nilpotent Lie group $N$. Then any compact set of $N$ is contained in a compact C-convex P-full subset of $N$.

Proof. The proof is by double induction on the dimension of $N$ and the degree of $P$, the assertion being trivial if the dimension of $N$ or the degree of $P$ is $\leqq 1$. We, therefore, suppose the theorem true whenever the dimension of the nilpotent group is $\langle n=\operatorname{dim} N$ or the degree of the operator is $\langle p=$ degree $P$.

If $Z$ is a one parameter central subgroup of $N$, the action of $P$ on $Z$-invariant functions gives rise to a differential operator $\tilde{P}$ on $N / Z$ satisfying $P f(x)$ $=\tilde{P} \widetilde{f}(\pi(x))$ whenever $f(x)=\breve{f}(\pi(x))$ where $\check{f}$ is a function on $N / Z$ and $\pi$ : $N \rightarrow N / Z$ is the natural projection. If $\tilde{P} \equiv 0$ it follows from lemma 1 that $P=z \circ P_{1}$ where $z$ is a generator of the Lie algebra of $Z$ and $P_{1}$ is a biinvariant operator on $N$. Since degree $P_{1}=p-1$ any compact set of $N$ is contained in a $P_{1}$-full compact $C$-convex subset $K$ of $N$ which is also $z$-full since this is the case for any $C$ convex subset of $N$. Now if $P f=u$ where $f$ and $u$ are compactly supported functions on $N$ with supp $u \subset K$, then $P f=z \circ P_{1} f=u$ so $P_{1} f$ is supported in $K$ since $K$ is $z$-full and $f$ is supported in $K$ since $K$ is $P_{1}$-full. Thus the induction is valid whenever $P$ annihilates all $Z$-invariant functions. Thus we can assume that whenever $Z$ is a one-parameter central subgroup of $N$, the differential operator $\tilde{P}$ on $N / Z$ induced by the action of $P$ on $Z$-invariant functions is non-zero and, therefore, by inductive hypothesis that any compact subset of $N / Z$ is contained in a $\tilde{P}$-full compact $C$-convex subset of $N / Z$.

The remainder of the proof is divided into two cases, viz.

Case 1. The center of $N$ has dimension 1 .

Case 2 . The center of $N$ has dimension $\geqq 2$. 
We deal with Case 1 first. Let $Z$ be the center of $N$, and let $z$ be a generator of the Lie algebra of $Z$. Since the center of $N / Z$ is non-trivial, we can find a vector $y \in \mathfrak{N}$, the Lie algebra of $N$ such that for all $x \in \mathfrak{N}$, we have $[y, x]=\phi(x) z$ where $\phi$ is a non-zero linear functional on $\mathfrak{N}$. Also $\left[y\left[x_{1}, x_{2}\right]\right]=\left[\left[y, x_{1}\right] x_{2}\right]$ $+\left[x_{1},\left[y, x_{2}\right]\right]=\left[\phi\left(x_{1}\right) z, x_{2}\right]+\left[x_{1}, \phi\left(x_{2}\right) z\right]=0$ so $\phi\left(\left[x_{1}, x_{2}\right]\right)=0$ and $\phi$ vanishes on the derived algebra of $\mathfrak{N}$. The kernel $\mathfrak{M}$ of $\phi$ is, therefore, a codimension one ideal of $\mathfrak{N}$ and we let $M=\exp \mathfrak{M}$ which is a simply connected nilpotent Lie subgroup of $N$ with Lie algebra $\mathfrak{M}$. We pick $w \in \mathfrak{N}$ with $\phi(\omega)=1$. Let $i: U(\mathfrak{M})$ $\rightarrow U(\mathfrak{N})$ be the inclusion of envelopping algebras induced by the inclusion of $\mathfrak{M}$ in $\mathfrak{N}$.

By the Poincare-Birkhoff-Witt theorem we can write $P$ uniquely as $P$ $=w^{k} \circ i\left(p_{0}\right)+w^{k-1} \circ i\left(p_{1}\right)+\cdots+w \circ i\left(p_{k-1}\right)+i\left(p_{k}\right)$ where the $p_{i}$ 's are elements of $U(\mathfrak{M})$ then

$$
0=[y, P]=\left[k \omega^{k-1} \circ i\left(p_{0}\right)+(k-1) \omega^{k-2} \circ i\left(p_{1}\right)+\cdots+i\left(p_{k-1}\right)\right] \circ z .
$$

This implies, again by the Poincaré-Birkhoff-Witt theorem that $0=p_{0}=p_{1}$ $=\cdots=p_{k-1}$ and, therefore, that $P=i\left(p_{k}\right)$. It follows that any subset $S$ of $N$ such that $x^{-1}(S \cap x M)$ is a $p_{k}$-full subset of $M$ for all $x$ is a $P$-full subset of $N$. Furthermore, since the center of $N$ is contained in $M$, if $x^{-1}(S \cap x M)$ is a $C$ convex subset of $M$ for all $x \in N$, then $S$ is a $C$-convex subset of $N$.

We pick a continuous $M$-equivariant projection $\psi: N \rightarrow M$ for instance

$$
\psi(x)=x[\exp \phi(-\log x) \omega] .
$$

Now let $K$ be a compact subset of $N$. By inductive hypothesis $\psi(K)$ is contained in a compact $C$-convex $p_{k}$-full subset $L$ of $M$. Also $\phi(\log K)$ is contained in a compact connected interval $I$ of $\mathbb{R}$. Now $\exp \phi^{-1}(I) \cap \psi^{-1}(L)$ is a compact $C$-convex $P$-full subset of $N$. This completes the proof of case 1 .

Case 2. The center of $N$ has dimension greater than 1 . Let $z_{1}$ and $z_{2}$ be vectors in the center of $\mathfrak{N}$ which are orthonormal for a Euclidean metric $\rho$ on $\mathfrak{N}$. Let $Z_{1}$ $=\exp \mathbb{R} z_{1}$ respectively $Z_{2}=\exp \mathbb{R} z_{2}$, and let $\pi_{1}$ respectively $\pi_{2}$ be the projections of $N$ on $N / Z_{1}$, respectively $N / Z_{2}$. Also let $P_{1}$ respectively $P_{2}$ be the differential operators on $N / Z_{1}$ respectively $N / Z_{2}$ induced by the action of $P$ on $Z_{1}$-invariant respectively $Z_{2}$-invariant functions on $N$. We can assume that neither $P_{1}$ nor $P_{2}$ is the zero operator. Let $K$ be a compact subset of $N$. Then $\pi_{1}(K)$ and $\pi_{2}(K)$ are compact subsets of $N / Z_{1}$ and $N / Z_{2}$ and by inductive hypothesis we can choose $F_{1} \supset \pi_{1}(K)$ and $F_{2} \supset \pi_{2}(K)$ such that $F_{i}$ is a $P_{i}$-full compact $C$-convex subset of $N / Z_{i}$. Then $\pi_{i}^{-1}\left(F_{i}\right)$ is a $C$-convex $P$-full subset of $N$ for $i=1,2$ by Proposition 1 and, therefore, $Q=\pi_{1}^{-1}\left(F_{1}\right) \cap \pi_{2}^{-1}\left(F_{2}\right)$ is a $C$-convex closed $P$-full subset of $N$ containing $K$.

We assert $Q$ is compact, or equivalently that $\log Q$ is compact. Let $\rho_{1}$ and $\rho_{2}$ be the Euclidean metrics induced by $\rho$ on $\mathfrak{N}_{1} \cong z_{1}^{\perp}$ and $\mathfrak{N}_{2} \cong z_{2}^{\perp}$, the Lie algebras of $N / Z_{1}$ and $N / Z_{2}$. We can find a real number $r$ such that the $\rho_{i}$ distance of $\log F_{i}$ from the origin of $\mathfrak{N}_{i}$ is $\leqq r$ for $i=1,2$. Then if $v \in \log \left(\pi_{1}^{-1}\left(F_{1}\right) \cap \pi_{2}^{-1}\left(F_{2}\right)\right)$ we have $\rho\left(v, \mathbb{R} x_{1}\right) \leqq r$ and $\rho\left(v, \mathbb{R} x_{2}\right) \leqq r$ so we can choose $t_{1}$ and $t_{2}$ such that $\rho\left(v, t_{1} z_{1}\right) \leqq r$ and $\rho\left(v, t_{2} z_{2}\right) \leqq r$. Then $\rho\left(t_{1} z_{1}, t_{2} z_{2}\right) \leqq 2 r$ so $\sqrt{t_{1}^{2}+t_{2}^{2}} \leqq 2 r$ so 
$t_{1}^{2} \leqq 4 r^{2}$ and $\rho\left(t_{1} z_{1}, 0\right)=\left|t_{1}\right| \leqq 2 r$. It follows that $\rho(v, 0) \leqq 3 r$ for all $v \in \log \left(\pi_{1}^{-1}\left(F_{1}\right) \cap \pi_{2}^{-1}\left(F_{2}\right)\right)$. Thus $\log \left(\pi_{1}^{-1}\left(F_{1}\right) \cap \pi_{2}^{-1}\left(F_{2}\right)\right)$ is a closed bounded subset of $\mathfrak{N}$ and, therefore, compact. Therefore, $\pi_{1}^{-1}\left(F_{1}\right) \cap \pi_{2}^{-1}\left(F_{2}\right)$ is a compact $C$ convex $P$-full subset of $N$ containing $K$. This completes the inductive step in Case 2 and concludes the proof of the theorem.

Corollary. If $K$ is any compact set in $N$, then there is a compact set $L$ such that whenever $P u=f$ is a distribution supported in $K$ and $u$ is a distribution of compact support on $N$, then the support of $u$ is contained in $L$.

Proof. This follows immediately from the theorem upon convoluting with a smooth approximate identity of $N$. Here $L$ can be any compact $P$-full set containing a compact neighborhood of $K$.

Theorem 2. Any non-zero biinvariant differential operator on a connected simply connected nilpotent Lie group is globally solvable.

Proof. Semi-global solvability of such operators is contained in results of Rais [8] or Rouvière [9]. But by theorem 1.9 in the book of Trèves [11], global solvability follows from semi-global solvability and the $P$-convexity result proved above.

\section{References}

1. Cerezo, A., Rouvière, F.: Résolubilité locale d'un opérateur differentiel invariant du premier ordre. Annales Scientifiques de l'Ecole Normale Supérieure 4, 21-30 (1971)

2. Cerezo, A., Rouvière, F.: Opérateurs différentiels invariants sur un groupe de Lie. Séminaire Goulaouic-Schwartz 1972-3, École Polytechnique, Paris

3. Duflo, M.: Opérateurs différentiels bi-invariants sur un groupe de Lie. To appear

4. Duflo, M., Rais, M.: Sur l'analyse harmonique sur les groupes de Lie résolubles. Annales Scientifiques de l'Ecole Normale Supérieure 9, 107-144 (1976)

5. Helgason, S.: Differential Geometry and Symmetric Spaces. New York: Academic Press 1962

6. Helgason, S.: The surjectivity of invariant differential operators on symmetric spaces. I. Annals of Mathematics 98, 451-479 (1973)

7. Hochschild, G.P.: The Structure of Lie Groups. San Francisco: Holden Day 1965

8. Rais, M.: Solutions élémentaires des operateurs différentiels bi-invariants sur un groupe de Lie nilpotent. Comptes Rendus de l'Académie des Sciences, Paris 273, 495-498 (1971)

9. Rouvière, F.: Sur la résolubilité locale des opérateurs biinvariants. Annali Scuola Normale Superiore-Pisa 3, 231-244 (1976)

10. Trèves, F.: Lectures on Linear Partial Differential Equations. Instituto de Matematica Pura e Aplicada, Rio de Janeiro, 1961

11. Trèves, F.: Linear Partial Differential Equations with Constant Coefficients. New York: Gordon and Breach 1966 